\title{
Optical View of a Merging Galaxy Cluster: Abell 2254
}

\author{
Ebru Aktekin Çalışkan ${ }^{1 *}$ \\ 1* Süleyman Demirel University, Faculty of Art and Science, Department of Physics, Isparta, Turkey, (ORCID: 0000-0002-5904-4580), ebrucaliskan@sdu.edu.tr
}

(First received 3 October 2021 and in final form 10 December 2021)

(DOI: 10.31590/ejosat.1003968)

ATIF/REFERENCE: Aktekin Caliskan, E. (2021). Optical View of a Merging Galaxy Cluster: Abell 2254. European Journal of Science and Technology, (31), 434-440.

\begin{abstract}
We present here the optical observations of a merging galaxy cluster $(\mathrm{z}=0.178)$ Abell 2254 (A2254) observed by the TÜBİTAKRTT150 Telescope in Antalya, Turkey. Its integrated CCD imaging results are reported. $B, V$, and $R$ brightness values are revealed for each member of the cluster, for the first time. We present the color magnitude diagram of A2254 for all galaxies. We found that the number of red galaxies is more than blue galaxies. We have obtained the galaxy luminosity function (GLF) using the $B$ band data. Here, we aim to analyze the effects of major cluster mergers on galaxy properties for better understanding. We have seen that the GLF of Abell 2254 can be fitted with a single Schechter function in the $B$ band. The best-fitting parameters of the Schechter function are the characteristic absolute magnitude in the $B$ band $M_{B}^{*}=19.67_{-0.96}^{+0.84} \mathrm{mag}$, and the slope at the faint end of the GLF $\alpha=-1.299_{-0.34}^{+0.23}$ for galaxies.
\end{abstract}

Keywords: Galaxies: clusters: individual: Abell 2254, galaxies: clusters. Galaxies: photometry, Luminosity function-galaxiesstatistics.

\section{Birleşen Galaksi Kümesine Optik Bakış: Abell 2254}

$\ddot{O} \mathbf{z}$

Bu çalışmada, Antalya, Türkiye'de TÜBİTAK-RTT150 Teleskopu tarafından gözlemlenen birleşen gökada kümesinin (z=0.178) Abell 2254 (A2254) optik gözlemleri sunulmuştur. Birleştirilmiş CCD görüntü sonuçları rapor edilmiştir. İlk kez kümenin her bir üyesi için $B, V$ ve $R$ parlaklık değerleri belirlenmiştir. Kümeye ait tüm galaksiler için renk parlaklık şeması sunulmuştur. Kırmızı galaksilerin sayısının mavi galaksilerden daha fazla olduğu sonucunu bulduk. $B$ band verilerini kullanarak gökada parlaklık fonksiyonunu (GLF) elde ettik. Burada amaç büyük küme birleşmesinin galaksi özellikleri üzerindeki etkilerinin daha iyi anlaşılmasıdır. Abell 2254'ün GLF'sinin $B$ bandında bir Schechter fonksiyonu ile uyduğu görüldü. Schechter fonksiyonunun en uygun parametreleri, $B$ bandındaki karakteristik mutlak büyüklük $M_{B}^{*}=19.67_{-0.96}^{+0.84}$ mag ve sönük uçtaki eğim $\alpha=-1.29_{-0.34}^{+0.23}$ bulunmuştur.

Anahtar Kelimeler: Galaksiler: kümeler: Özel: Abell 2254, Galaksiler: Kümeler: Fotometri, Işınım gücü fonksiyonu-galaksileristatistik.

\footnotetext{
* Corresponding Author: ebrucaliskan@sdu.edu.tr
} 


\section{Introduction}

Galaxy clusters are the largest structures detected to be covering many galaxies $(>50)$ and the hot ionized gas in hydrostatic equilibrium filling the intra-cluster medium (ICM) and the dark matter. This hot gas in the cluster has electron density values in the range of $10^{-4}-10^{-2} \mathrm{~cm}^{-3}$ with a temperature range of $10^{7}-10^{8} \mathrm{~K}$. Their radius is detected to be between 1 and $2 \mathrm{Mpc}$ (with Hubble constant of $\mathrm{H}_{0}=70 \mathrm{~km} \mathrm{~s}^{-1} \mathrm{Mpc}^{-1}$ ). Their emission is detected to be thermal Bremsstrahlung (see e.g. Sarazin, 1992, Sarazin, 1988, Fabian1988, Sarazin, 1986).

Optical observations of early-type galaxies provide important clues about the physical processes that influenced the formation and evolution of these galaxies. Baum (1959) explained that as galaxies get brighter, the field elliptical colors become redder. In the following years, the relationship between increasing luminosity and increasing metallicity was interpreted as the color-magnitude relation (CMR) (Caglar \& Hudaverdi 2017, Gallazzi et al. 2006, Kodama et al. 1999; Kodama \& Arimoto 1997; Worthey 1994; Faber 1973).

Detailed studies of galaxy clusters at optical wavelengths allow us to investigate the distribution of the number of galaxies in the unit volume and unit luminosity range through the study of the galaxy luminosity function (GLF) (Feng et al. 2014). The first study on the GLF was published in 1974 by Press and Schechter (1974) simply presented the hierarchical clustering of a galaxy cluster in terms of mass distribution. The empirical formula of the galaxy luminosity function was proposed by Schechter (1976). Schechter function

$$
\Phi(M) d M=\Phi^{*} 10^{-0,4(\alpha+1)\left(M-M^{*}\right)} \exp \left[-10^{-0,4\left(M-M^{*}\right)}\right] d M
$$

in which:

$\Phi(\mathrm{M}) \mathrm{dM}$ : the number of galaxies with absolute magnitudes in the range from $\mathrm{M}+\mathrm{dM}$ (per unit volume)

$\Phi^{*}$ : a normalization factor that defines the overall density of galaxies (number per cubic Mpc),

\section{$\mathrm{M}^{*}$ : the characteristic absolute magnitude.}

Studies of normal galaxy clusters and merging clusters have been done at all wavelengths (e.g. Boué et al. 2008, Barkhouse et al. 2007, Adami et al. 2007, De Propris et al. 2003). Distance clusters of galaxies have also been explored by Andreon (2001, z $=0.3$ ), Massarotti et al. (2003, $z=0.64)$, and Drory et al. (2003, $\mathrm{z} \sim 1.2)$. The relative distributions and shapes of bright and faint galaxies in clusters are affected by mergers. GLFs are also useful to characterize these effects. Although many bright galaxies are seen, the GLFs of some merging clusters of galaxies have been shown dips and wiggles (see e.g. Durret et al. 2011 and references therein). A large number of spectroscopic galaxy redshifts can make becomes possible the dynamical properties and estimate the mass of the cluster, as well as characterize substructures, if any. From the X-ray data, temperature and metallicity maps of the X-ray gas can serve as complements to probe the substructures. Numerical simulations of optical and Xray results can provide information on cluster formation (see eg Durret et al. 2011 and references therein).
Abell 2254 (A2254), is reported by Crawford et al. (1995) with a red-shift of $z=0.178$. This cluster has an enrichment class of 2 as described by (Abell et al. 1989) and is known to be a rich galaxy cluster. Ebeling et al. (1996) reported that it as an $\mathrm{X}$-ray bright cluster with $L_{X}=7.19 \times 10^{44} \mathrm{ergs} \mathrm{s}^{-1}$ in the energy range of $(0.1-2.4) \mathrm{keV}$ as a result of ROSAT observations. Considering both radio and X-ray emission, it is seen that it has a very clumpy and irregular structure. The A2254 structure is known to be the irregular structure of a dynamically young cluster, in which fragments with different morphology both in Xray and radio morphology merge (Schindler \& Müller 1993).

Girardi et al. (2011) reported the internal dynamics of A2254. Their analysis was based on redshift data for 128 galaxies. They combined galaxy velocities and positions to select 110 cluster galaxies and analyzed their internal dynamics. They used optical (new g', r', i' photometric data from the Isaac Newton Telescope and Subaru Archive V, i' photometric data) and X-ray data (XMM-Newton) in their work. They have estimated the cluster redshift to be $\mathrm{z}=0.177$, a high line-of-sight velocity dispersion of $\sigma_{\mathrm{v}} \sim 1350 \mathrm{~km} \mathrm{~s}^{-1}$, and the X-ray temperature of $\mathrm{kT} \sim 6.4 \mathrm{keV}$. Their optical and X-ray analyses revealed complex dynamical activity in both electromagnetic windows. Their X-ray morphological analysis with $X M M$ Newton data confirmed that A2254 is a dynamically disturbed cluster. Their X-ray studies revealed that the cluster had to be elongated toward the eastern direction, which has shown a good agreement with a merger in the post-core-crossing phase. Their optical and X-ray analyses showed that the main system was a non-relaxed structure, indicating north-south as a possible direction for a past accretion. They concluded that A2254, for its mass and merging structure, suits well among the typical clusters with radio-halos (Girardi et al. 2011).

Girardi et al. (2012) also reported that the X-ray temperature of $\mathrm{kT} \sim 6.4 \mathrm{keV}$. The main system was interpreted as likely substructured. The X-ray mechanisms of early-type galaxies can be understood as follows: either they are X-ray emitted systems via accretion disk around the compact objects which are low mass binaries and almost most of them are thought to produce their Xrays by accreting material onto the disk, and/or the compact objects which are supermassive black holes in the Active Galactic Nuclei (AGN). Another alternative could be due to the existence of a very hot gas of temperature $\left(\sim 10^{7} \mathrm{~K}\right)$ in the halo of the interstellar medium where X-rays come from Smith et al. (2001). Expected $\log \left(L_{X}\right) \geq 40$ ergs $\mathrm{s}^{-1}$ criteria for being a possible AGN is reported by Blanton et al. (2001), Randall et al. (2006). Ebeling et al. (1996) reported it as an X-ray bright cluster with $L_{X}=7.19 \times 10^{44} \mathrm{ergs} \mathrm{s}^{-1}$ in the energy range of (0.12.4) $\mathrm{keV}$ as a result of ROSAT observations.

The X-ray studies of local early-type galaxies have correlate the total X-ray luminosity is associated with optical luminosity (e.g. Ellis \& O’Sullivan, 2006, O’Sullivan et al, 2001). Consequently, the $L_{X} / L_{B}$ luminosity ratio demonstrates the stellar velocity dispersions of a defined galaxy (traced by $L_{X}$ ) and the stellar mass (traced by $L_{B}$ ) of that galaxy (Lehmer et al, 2007, Mahdavi \& Geller, 2001). Canizares et al. (1987) defined X-ray to optical luminosity correlation as $L_{X} \propto L_{B}^{1.6-2.3}$ for optically bright sources. The correlation for optically faint galaxies is observed to be rather linear, $L_{X} \propto L_{B}$ (e.g. Gilfanov 2004). 
In this work, we presented our optical observations and data reduction of A2254 in Section 2, and our discussion and conclusions in Section 3 and 4, respectively.

\section{Material and Method}

\subsection{Optical Observations and Data Reduction}

The optical imaging observations of A2254 took place on the 11th of September 2015 with a 1.5-m Russian-TurkishTelescope $\left(\right.$ RTT150) ${ }^{\dagger}$ located in Antalya, Turkey. The observations are performed with the RTT150. During the observations, the seeing varied 1.8 arcsec. The optical images were obtained using the TFOSC instrument at the Cassegrain focus. The $2048 \times 2048$ back illuminated CCD has $15 \mu \mathrm{m}^{2}$ pixel size, and $13.5 \times 13.5 \mathrm{arcmin}^{2}$ field of view.

The filters $B, V$, and $R$ were used with exposure times of $3 \times 900 \mathrm{~s}$ for individual filter imaging observations. Exposure times and filter properties for imaging observations can be seen in Table 1.

Table 1. Log of Observations of A2254

\begin{tabular}{|l|l|l|l|l|}
\hline $\begin{array}{l}\text { Observation } \\
\text { Date }\end{array}$ & $\begin{array}{l}\text { Exposure } \\
\text { Times } \\
(\mathrm{s})\end{array}$ & Filter & $\begin{array}{l}\text { FWHM } \\
(\mathrm{nm})\end{array}$ & $\begin{array}{l}\lambda \text { cent. } \\
(\mathrm{nm})\end{array}$ \\
\hline $11 / 09 / 2015$ & $900 \times 3$ & Bessel B & 114 & 433 \\
\hline $11 / 09 / 2015$ & $900 \times 3$ & Bessel V & 100 & 519 \\
\hline $11 / 09 / 2015$ & $900 \times 3$ & Bessel R & 128 & 600 \\
\hline
\end{tabular}

The reduction of raw data was processed by using an Image Reduction and Analysis Facility (IRAF). The imaging tool of DS9 was used for imaging data reduction and analysis in which the bias corrections, over-scan at the field, and cosmic ray elimination were included by making the use of the IRAF CCDPROC package. To inspect the locations of the source positions, we used the USNO- A2.0 catalog of astrometric standards by Monet et al. (1998). Figure 1 shows the A2254 optical RTT150 image in the Bessel $R$ filter.
Figure 1. Integrated (total) Bessel R filter image of A2254. Red circles are the galaxies detected in the cluster while green circles are the ones that are not detected in the cluster itself, but rather detected along the line of sight that is so-called non-member galaxies

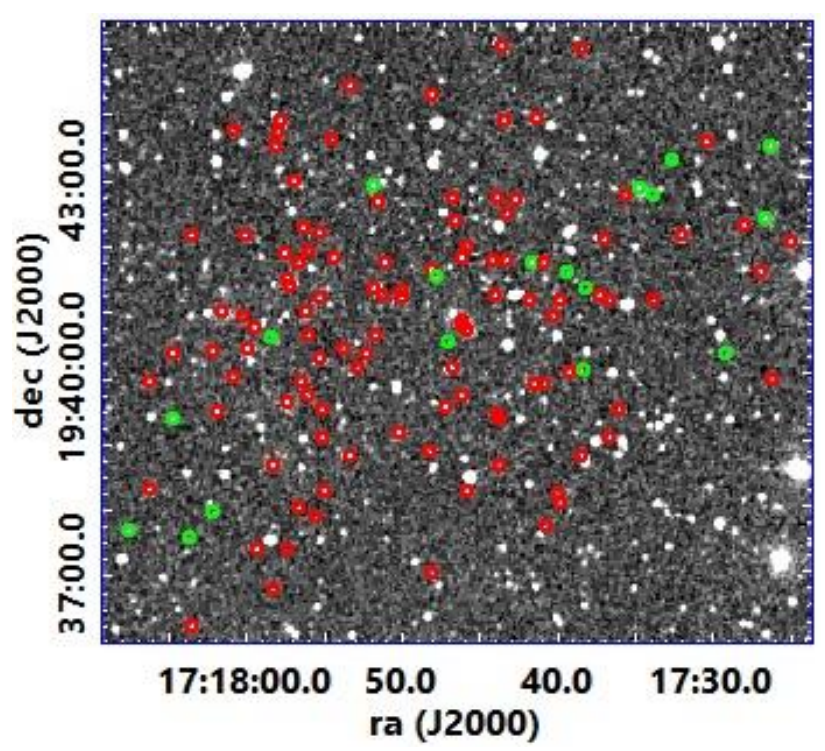

After completing the pre-reduction tasks explained above, we obtained the total-integrated imaging data for each filter and calculated the magnitudes of the galaxies with the help of their aperture values. Galaxies are detected and galaxy catalogs were compiled using SExtractor (Bertin \& Arnouts, 1996). This is presented in Table 2, where we have also used the identified member galaxies in A2254 by Girardi et al. (2011).

We made the detections with the $R$ filter because it is more sensitive. Measurements were made with $B$-band with the double image mode. We accepted the minimum required 5 pixels required and a detection threshold of 1.5 sigmas for galaxy detection. The galaxies are detected with the SExtractor software (Bertin \& Arnouts 1996). Total magnitudes have been calculated in the Vega system and all magnitudes corrected for galactic extinction. All galaxies are listed in Table 2, in its column (IDm) are found to be in very good agreement with the positions of the galaxies reported by Girardi et al. (2011). We derived the magnitudes of all galaxies in A2254 for member and nonmember ones using $B, V$, and $R$ filters.

\footnotetext{
${ }^{\dagger}$ Specifications of RTT150 and TÜBİTAK Faint Object

Spectrometer and Camera (TFOSC) are available at http://www.tug.tubitak.gov.tr

${ }^{\ddagger}$ Developed by USA National Optical Astronomical Observatories (NOAO) \/https://iraf-community.github.io/
} 
Table 2. A2254 cluster member galaxies with their positions detected in this work and their magnitudes obtained for filters $B$, $V$, and R. Column (ID) shows all galaxies observed during our observations including the non-members but in the line of sight, while column (IDm) shows the galaxies detected as members) in A2254. All galaxies are listed in columns (ID and IDm) are found to be in very good agreement with the positions of the galaxies reported by (Girardi et al. 2011).

\begin{tabular}{|c|c|c|c|c|c|c|}
\hline ID & $\mathrm{IDm}$ & $\begin{array}{l}\text { ra (J2000) } \\
(\mathrm{h} \mathrm{m} \mathrm{s})\end{array}$ & $\begin{array}{l}\text { dec (J2000) } \\
(\mathrm{O} / \mathrm{\prime})\end{array}$ & $\begin{array}{l}B \\
(\mathrm{mag})\end{array}$ & $\begin{array}{l}V \\
(\mathrm{mag})\end{array}$ & $\begin{array}{l}R \\
(\mathrm{mag})\end{array}$ \\
\hline 1 & 1 & $17: 17: 24.62$ & $+19: 41: 59.6$ & 17.86 & 17.56 & 16.56 \\
\hline 2 & 2 & $17: 17: 25.92$ & $+19: 39: 54.7$ & 20.30 & 19.39 & 18.99 \\
\hline 3 & & $17: 17: 25.97$ & $+19: 43: 25.3$ & 18.09 & 17.15 & 16.49 \\
\hline 4 & & $17: 17: 26.26$ & $+19: 42: 20.5$ & 18.58 & 17.74 & 17.27 \\
\hline 5 & 3 & $17: 17: 26.59$ & $+19: 41: 31.6$ & 18.66 & 18.06 & 17.82 \\
\hline 6 & 4 & $17: 17: 27.62$ & $+19: 42: 14.8$ & 18.64 & 18.06 & 17.24 \\
\hline 7 & & $17: 17: 28.94$ & $+19: 40: 18.1$ & 21.08 & 19.98 & 19.34 \\
\hline 8 & 5 & $17: 17: 30.10$ & $+19: 43: 30.0$ & 19.53 & 18.48 & 18.18 \\
\hline 9 & 6 & $17: 17: 31.68$ & $+19: 42: 06.1$ & 19.16 & 18.00 & 17.84 \\
\hline 10 & & $17: 17: 32.35$ & $+19: 43: 13.4$ & 20.19 & 19.52 & 19.12 \\
\hline 11 & 7 & $17: 17: 33.53$ & $+19: 41: 07.1$ & 19.47 & 19.03 & 18.80 \\
\hline 12 & & $17: 17: 33.55$ & $+19: 42: 42.1$ & 20.19 & 19.47 & 18.99 \\
\hline 13 & & $17: 17: 34.37$ & $+19: 42: 48.6$ & 17.25 & 16.72 & 16.08 \\
\hline 14 & 8 & $17: 17: 35.35$ & $+19: 42: 42.8$ & 19.20 & 18.92 & 18.18 \\
\hline 15 & 9 & $17: 17: 35.95$ & $+19: 39: 27.4$ & 19.96 & 19.43 & 18.82 \\
\hline 16 & 10 & $17: 17: 36.43$ & $+19: 41: 07.8$ & 19.84 & & 18.63 \\
\hline 17 & 11 & $17: 17: 36.46$ & $+19: 39: 02.2$ & 19.17 & & 18.24 \\
\hline 18 & 12 & $17: 17: 36.74$ & $+19: 42: 03.6$ & 20.72 & & 19.43 \\
\hline 19 & 13 & $17: 17: 37.03$ & $+19: 41: 11.4$ & 19.27 & & 18.40 \\
\hline 20 & & $17: 17: 37.87$ & $+19: 41: 17.9$ & 20.92 & & 19.39 \\
\hline 21 & & $17: 17: 38.04$ & $+19: 40: 04.4$ & 19.70 & 19.27 & 18.25 \\
\hline 22 & 14 & $17: 17: 38.04$ & $+19: 44: 55.7$ & 20.19 & 19.31 & 18.90 \\
\hline 23 & 15 & $17: 17: 38.21$ & $+19: 40: 03.7$ & 20.90 & 20.81 & 19.80 \\
\hline 24 & 16 & $17: 17: 38.26$ & $+19: 38: 46.3$ & 20.61 & 9.98 & 19.34 \\
\hline 25 & 17 & $17: 17: 38.95$ & $+19: 40: 01.6$ & 19.44 & 8.87 & 18.11 \\
\hline 26 & & $17: 17: 39.10$ & $9: 41: 33.0$ & .64 & 9.79 & 19.02 \\
\hline 27 & 18 & $17: 17: 39.65$ & $9: 41: 06.7$ & 0.27 & 9.27 & 18.90 \\
\hline 28 & 19 & $17: 17: 39.70$ & $9: 38: 02.8$ & .37 & & \\
\hline 29 & 20 & $17: 17: 39.84$ & $9: 38: 10.7$ & .58 & .52 & 9.43 \\
\hline 30 & 21 & $17: 17: 39.84$ & $9: 38: 10.7$ & .22 & & 3.99 \\
\hline 31 & 22 & $17: 17: 40.03$ & $19: 40: 52.0$ & 3.92 & & 7.63 \\
\hline 32 & 23 & $17: 17: 40.63$ & $9: 37: 42.6$ & .09 & & .30 \\
\hline 33 & 24 & $7: 17: 40.66$ & $: 40.9$ & .50 & & .11 \\
\hline 34 & 25 & 7:40.68 & 52.2 & 66 & & 34 \\
\hline 35 & 26 & & 53.8 & 19.83 & & 18.90 \\
\hline 36 & 27 & 1.33 & $: 52.2$ & 19.66 & 18.89 & 18.82 \\
\hline 37 & & 41.38 & $+19: 41: 42.0$ & 18.26 & 18.06 & 17.36 \\
\hline 38 & 28 & $17: 17: 41.57$ & $+19: 41: 07.4$ & 18.69 & 17.97 & 17.55 \\
\hline 39 & 29 & $17: 17: 42.34$ & $+19: 42: 39.2$ & 17.72 & 16.97 & 16.74 \\
\hline 40 & 30 & $17: 17: 43.01$ & $+19: 42: 26.3$ & 18.86 & 17.97 & 17.47 \\
\hline 41 & 31 & 17:17:43.06 & $+19: 41: 44.2$ & 18.77 & 17.97 & 17.66 \\
\hline 42 & 32 & 17:17:43.06 & 19:43:51.6 & 18.79 & 17.66 & 17.56 \\
\hline 43 & 33 & $17: 17: 43.20$ & $+19: 44: 59.6$ & 18.81 & 8.27 & 17.74 \\
\hline 44 & 34 & 17:17:43.61 & $19: 39: 20.5$ & 19.15 & 18.68 & 17.81 \\
\hline 45 & 35 & $17: 17: 43.61$ & $19: 42: 42.1$ & 19.40 & 18.68 & 18.04 \\
\hline 46 & 36 & $17: 17: 43.63$ & $+19: 38: 37.7$ & 20.89 & 20.81 & 19.87 \\
\hline 47 & 37 & $17: 17: 43.70$ & $+19: 39: 24.8$ & 20.72 & 20.43 & 19.63 \\
\hline 48 & 38 & $17: 17: 43.80$ & $+19: 41: 12.1$ & 19.06 & 18.34 & 17.85 \\
\hline 49 & 39 & $17: 17: 43.87$ & $+19: 41: 43.4$ & 19.47 & 18.17 & 17.97 \\
\hline 50 & 40 & $17: 17: 45.53$ & $+19: 40: 40.1$ & 18.55 & 17.96 & 17.45 \\
\hline 51 & 41 & $17: 17: 45.62$ & $+19: 41: 57.1$ & 20.97 & 20.14 & 19.57 \\
\hline 52 & 42 & $17: 17: 45.65$ & $+19: 38: 13.6$ & 18.99 & 18.48 & 17.82 \\
\hline 53 & 43 & $17: 17: 45.86$ & $+19: 40: 48.4$ & 17.14 & 16.14 & 15.73 \\
\hline 54 & 44 & $17: 17: 45.86$ & $+19: 41: 47.4$ & 19.38 & 18.31 & 18.11 \\
\hline 55 & 45 & $17: 17: 45.86$ & $+19: 40: 45.8$ & 20.23 & 19.67 & 19.15 \\
\hline 56 & 46 & 17:17:45.91 & $+19: 39: 41.4$ & 19.04 & 18.41 & 17.63 \\
\hline 57 & 47 & $17: 17: 46.30$ & $+19: 42: 20.9$ & 19.93 & 19.23 & 18.61 \\
\hline 58 & 48 & $17: 17: 46.51$ & $+19: 42: 42.5$ & 20.26 & 19.31 & 19.12 \\
\hline
\end{tabular}

\begin{tabular}{|c|c|c|c|c|c|c|}
\hline ID & $\mathrm{IDm}$ & $\begin{array}{l}\text { ra (J2000) } \\
(\mathrm{h} \mathrm{m} \mathrm{s})\end{array}$ & $\begin{array}{l}\text { dec (J2000) } \\
(\mathrm{O} / \mathrm{II})\end{array}$ & $\begin{array}{l}B \\
(\mathrm{mag})\end{array}$ & $\begin{array}{l}V \\
\text { (mag) }\end{array}$ & $\begin{array}{l}R \\
(\mathrm{mag})\end{array}$ \\
\hline 59 & 49 & $17: 17: 46.58$ & $+19: 40: 05.9$ & 19.36 & 19.06 & 18.57 \\
\hline 60 & & $17: 17: 46.75$ & $+19: 40: 30.7$ & 18.96 & 18.39 & 18.18 \\
\hline 61 & 50 & $17: 17: 46.97$ & $+19: 39: 31.3$ & 18.38 & 18.02 & 16.99 \\
\hline 62 & & $17: 17: 47.59$ & $+19: 41: 29.4$ & 20.16 & 19.67 & 19.34 \\
\hline 63 & 51 & $17: 17: 47.69$ & $+19: 44: 15.4$ & 19.27 & 18.39 & 18.33 \\
\hline 64 & 52 & $17: 17: 47.78$ & $+19: 41: 35.9$ & 18.82 & 18.17 & 17.63 \\
\hline 65 & 53 & $17: 17: 47.95$ & $+19: 37: 00.8$ & 20.29 & 19.31 & 18.99 \\
\hline 66 & 54 & $17: 17: 48.02$ & $+19: 38: 51.4$ & 19.79 & 19.35 & 18.55 \\
\hline 67 & 55 & $17: 17: 49.78$ & $+19: 41: 10.7$ & 19.63 & 18.68 & 18.51 \\
\hline 68 & 56 & $17: 17: 49.82$ & $+19: 41: 18.2$ & 20.56 & 20.43 & 19.30 \\
\hline 69 & 57 & $17: 17: 50.02$ & $+19: 39: 07.6$ & 17.73 & 17.02 & 16.28 \\
\hline 70 & 58 & $17: 17: 50.81$ & $+19: 41: 43.4$ & 18.50 & 17.41 & 17.24 \\
\hline 71 & 59 & $17: 17: 50.95$ & $+19: 41: 12.5$ & 20.40 & 19.35 & 19.19 \\
\hline 72 & 60 & $17: 17: 51.22$ & $+19: 42: 38.5$ & 18.32 & 17.97 & 17.33 \\
\hline 73 & 61 & $17: 17: 51.48$ & $+19: 40: 36.1$ & 18.66 & 18.17 & 17.24 \\
\hline 74 & & $17: 17: 51.58$ & $+19: 42: 52.9$ & 18.09 & 17.15 & 16.60 \\
\hline 75 & 62 & $17: 17: 51.65$ & $+19: 41: 20.4$ & 19.99 & 18.87 & 18.65 \\
\hline 76 & 63 & $17: 17: 52.13$ & $+19: 40: 19.6$ & 18.64 & 18.50 & 17.28 \\
\hline 77 & 64 & $17: 17: 52.61$ & $+19: 40: 08.0$ & 19.59 & 18.92 & 18.18 \\
\hline 78 & 65 & $17: 17: 53.04$ & $+19: 44: 24.0$ & 19.40 & 18.30 & 18.04 \\
\hline 79 & 66 & $17: 17: 53.21$ & $+19: 38: 47.8$ & 18.27 & 18.10 & 17.01 \\
\hline 80 & 67 & $17: 17: 53.52$ & $+19: 40: 25.0$ & 20.02 & 18.92 & 18.72 \\
\hline 81 & 68 & $17: 17: 54.12$ & $+19: 41: 47.8$ & 19.05 & 18.31 & 17.72 \\
\hline 82 & 69 & $17: 17: 54.17$ & $+19: 43: 36.1$ & 20.62 & 20.67 & 19.39 \\
\hline 83 & 70 & $17: 17: 54.84$ & $+19: 38: 14.3$ & 19.29 & 18.68 & 18.24 \\
\hline 84 & 71 & $17: 17: 54.91$ & $+19: 39: 04.3$ & 19.77 & 18.98 & 18.40 \\
\hline 85 & 72 & $17: 17: 54.96$ & $+19: 39: 29.2$ & 19.85 & 19.67 & 19.22 \\
\hline 86 & 73 & $17: 17: 55.06$ & $+19: 40: 16.0$ & 18.31 & 17.84 & 16.99 \\
\hline 87 & 74 & $17: 17: 55.06$ & $+19: 42: 10.8$ & 19.86 & 19.03 & 18.42 \\
\hline 88 & 75 & $17: 17: 55.08$ & $+19: 41: 10.7$ & 19.08 & 18.59 & 17.63 \\
\hline 89 & 76 & $17: 17: 55.46$ & $+19: 37: 52.0$ & 20.71 & 20.43 & 19.48 \\
\hline 90 & 77 & $17: 17: 55.75$ & $+19: 41: 53.2$ & 20.61 & 19.85 & 19.57 \\
\hline 91 & 78 & $17: 17: 55.78$ & $+19: 39: 41.4$ & 19.83 & 18.92 & 18.51 \\
\hline 92 & 79 & $17: 17: 55.78$ & $+19: 40: 36.8$ & 19.01 & 18.52 & 18.33 \\
\hline 93 & 80 & $17: 17: 56.02$ & $+19: 40: 59.5$ & 18.56 & 17.89 & 17.25 \\
\hline 94 & 81 & $17: 17: 56.09$ & $+19: 42: 14.4$ & 20.30 & 19.79 & 19.30 \\
\hline 95 & 82 & $17: 17: 56.30$ & $+19: 39: 54.4$ & 19.27 & 18.39 & 17.92 \\
\hline 96 & 83 & $17: 17: 56.42$ & $+19: 41: 43.4$ & 19.67 & 19.06 & 18.51 \\
\hline 97 & 84 & $17: 17: 56.50$ & $+19: 37: 59.9$ & 19.74 & 19.27 & 18.40 \\
\hline 98 & 85 & $17: 17: 56.62$ & $+19: 42: 56.5$ & 18.99 & 18.39 & 17.63 \\
\hline 99 & 86 & $17: 17: 56.95$ & $+19: 41: 21.5$ & 19.59 & 18.89 & 18.33 \\
\hline 100 & 87 & $17: 17: 57.07$ & $+19: 41: 28.7$ & 18.35 & 17.32 & 17.00 \\
\hline 101 & 88 & $17: 17: 57.19$ & $+19: 37: 23.5$ & 20.51 & 19.79 & 19.39 \\
\hline 102 & 89 & $17: 17: 57.26$ & $+19: 39: 35.3$ & 17.55 & 16.59 & 16.24 \\
\hline 103 & 90 & $17: 17: 57.29$ & $+19: 41: 52.4$ & 18.51 & 17.73 & 17.16 \\
\hline 104 & 91 & $17: 17: 57.53$ & $+19: 43: 52.0$ & 18.73 & 17.65 & 17.38 \\
\hline 105 & 92 & $17: 17: 57.67$ & $+19: 43: 42.2$ & 19.80 & 19.16 & 18.72 \\
\hline 106 & 93 & $17: 17: 57.84$ & $+19: 43: 28.9$ & 18.72 & 18.30 & 18.04 \\
\hline 107 & 94 & $17: 17: 58.13$ & $+19: 38: 39.1$ & 18.22 & 17.58 & 17.21 \\
\hline 108 & 95 & $17: 17: 58.20$ & $+19: 36: 45.7$ & 20.31 & 19.31 & 18.99 \\
\hline 109 & & $17: 17: 58.20$ & $+19: 40: 36.5$ & 19.84 & 19.31 & 18.96 \\
\hline 110 & 96 & $17: 17: 59.21$ & $+19: 37: 23.2$ & 19.72 & 18.79 & 18.51 \\
\hline 111 & 97 & $17: 17: 59.21$ & $+19: 40: 45.1$ & 18.99 & 18.24 & 17.64 \\
\hline 112 & 98 & $17: 17: 59.69$ & $+19: 40: 25.7$ & 17.45 & 16.68 & 16.05 \\
\hline 113 & 99 & $17: 17: 59.83$ & $+19: 42: 09.0$ & 19.17 & 18.84 & 18.42 \\
\hline 114 & 100 & $17: 17: 59.98$ & $+19: 40: 54.1$ & 20.26 & 19.85 & 19.34 \\
\hline 115 & 101 & $17: 18: 00.58$ & $+19: 43: 44.8$ & 20.43 & 19.85 & 19.34 \\
\hline 116 & 102 & 17:18:00.67 & $+19: 40: 00.1$ & 20.33 & 19.57 & 18.96 \\
\hline 117 & 103 & $17: 18: 01.30$ & $+19: 40: 58.4$ & 17.34 & 16.52 & 16.07 \\
\hline 118 & 104 & $17: 18: 01.70$ & $+19: 39: 28.8$ & 18.75 & 17.93 & 17.40 \\
\hline 119 & 105 & $17: 18: 01.97$ & $+19: 40: 22.4$ & 18.90 & 18.27 & 17.63 \\
\hline 120 & & 17:18:01.99 & $+19: 37: 56.6$ & 19.39 & 19.03 & 18.45 \\
\hline 121 & 106 & $17: 18: 03.31$ & $+19: 42: 09.4$ & 19.46 & 18.84 & 18.18 \\
\hline 122 & 107 & 17:18:03.46 & $+19: 36: 14.0$ & 19.37 & 18.98 & 18.11 \\
\hline 123 & & $17: 18: 03.62$ & $+19: 37: 33.6$ & 20.64 & 19.62 & 19.30 \\
\hline 124 & 108 & $17: 18: 04.46$ & $+19: 40: 21.4$ & 18.97 & 19.67 & 18.68 \\
\hline 125 & & $17: 18: 04.61$ & $+19: 39: 22.3$ & 19.09 & 18.30 & 17.40 \\
\hline 126 & 109 & $17: 18: 06.00$ & $+19: 39: 55.1$ & 19.12 & 18.39 & 17.98 \\
\hline 127 & 110 & $17: 18: 06.05$ & $+19: 38: 17.5$ & 17.62 & 16.78 & 16.29 \\
\hline
\end{tabular}




\begin{tabular}{|l|l|l|l|l|l|l|}
\hline ID & IDm & $\begin{array}{l}\text { ra (J2000) } \\
(\mathrm{h} \mathrm{m} \mathrm{s})\end{array}$ & $\begin{array}{l}\operatorname{dec}(\mathrm{J} 2000) \\
(\mathrm{O} / \mathrm{I})\end{array}$ & $\begin{array}{l}B \\
(\mathrm{mag})\end{array}$ & $\begin{array}{l}V \\
(\mathrm{mag})\end{array}$ & $\begin{array}{l}R \\
(\mathrm{mag})\end{array}$ \\
\hline 128 & & $17: 18: 07.51$ & $+19: 37: 40.8$ & 19.22 & 18.48 & 18.04 \\
\hline
\end{tabular}

The $B$ - $R$ vs $R$ color-magnitude diagram is presented in Figure 2. Galaxies are divided into three groups in Figure 2: red, blue, and background galaxies. In this figure, red line is the best fit colormagnitude relation. When $B-V<B-V-0.4 \mathrm{mag}$, galaxies are getting bluer and these galaxies contain mainly star-forming galaxies, both in the cluster and in the field. When $B-V>B-V-$ $0.4 \mathrm{mag}$, galaxies are background galaxies.

Figure 2. The B-R vs $R$ color-magnitude diagram for the cluster A2254, divided into three groups: red, blue, and background galaxies

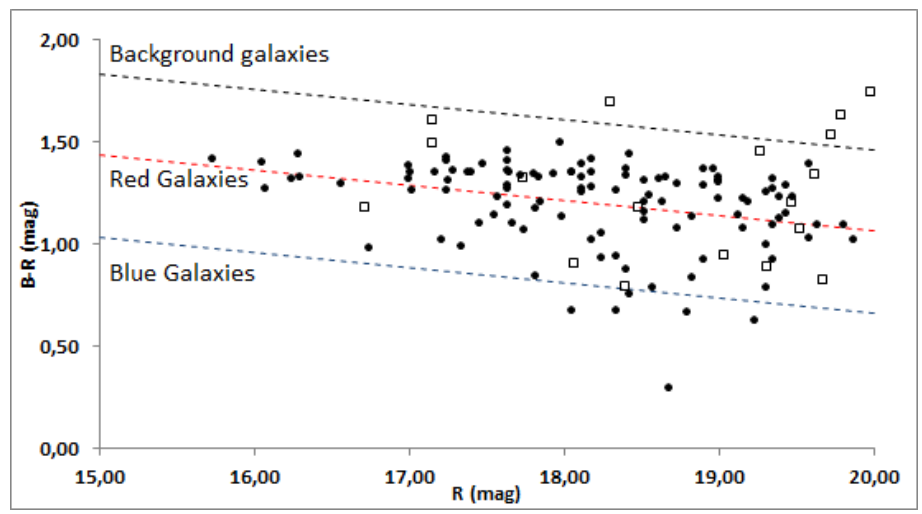

The resulting GLFs were calculated in bins of 0.5 mags as a function of absolute B magnitude, they are shown in Figure 3. We subtracted the background contribution using background galaxy counts.

Figure 3. Galaxy luminosity function in the B band for A2254. The continuous line represents the Schechter function.

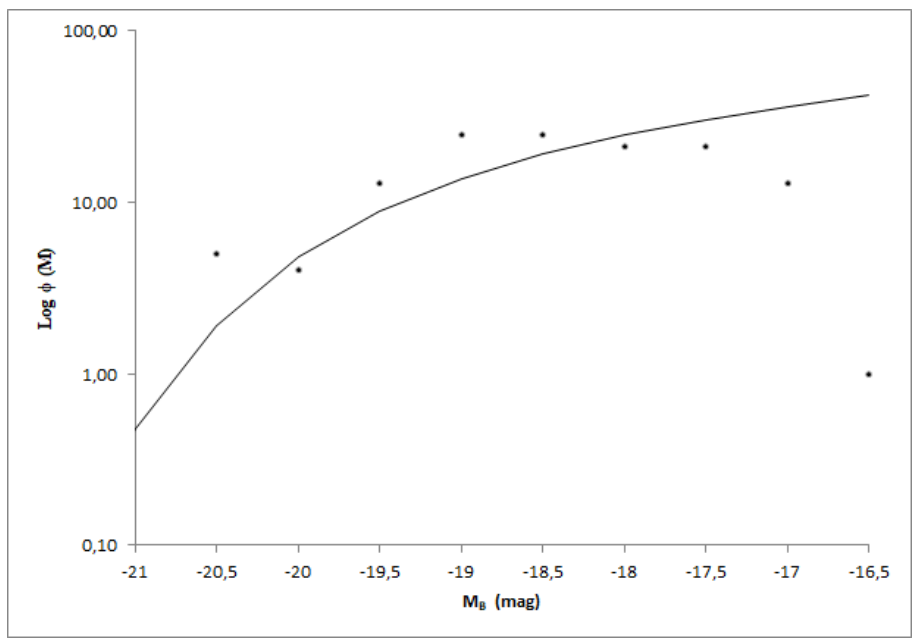

\section{Results and Discussion}

We have presented here the optical imaging observational results of a merging galaxy cluster A2254. In optical imaging search, we have used the Bessel $B, V$, and $R$ filters, for the first time.
In this study, 128 galaxies were identified as the main followers of cluster dynamics, which is in very good agreement with Girardi et al. (2011). For a better understanding of dynamical structure in A2254, faint member galaxies should also be taken into account. All galaxies are listed in Table 2. Member galaxies and galaxies reported by Girardi et al. (2011) have same position. We have derived the magnitudes of all galaxies in A2254 for member and non-member ones using $B, V$, and $R$ filters. Our Figure 1 is the Bessel R filter image of A2254 and Table 2 is the reported magnitudes for the whole galaxies located in $\mathrm{A} 2254$.

ID53 is located at the center of the cluster and is the brightest member of the cluster. Also, ID102, 112, and 117 have their brightness close to ID53 ( $<5$ mags) and these galaxies are located in the eastern part of the cluster. The cluster, as is known, is classified as "B", (binary) according to the Rood-Sastry morphological type (Struble \& Rood 1987).

ID53 has a bright companion (ID50). According to Subaru images (Girardi et al. 2011), this companion is a spiral galaxy with a large bulge and an arm directed toward the ID53 and characterized by emission spectral lines (e.g. [OII], $\mathrm{H} \beta$, and [OIII]). Although we have obtained the optical spectrum of ID53, it was not possible to distinguish between the emission lines of the two galaxies in the optical spectrum of ID53.

Our observed $L_{B}$ values are in the range of $0.77 \times 10^{9}-0.38$ x $10^{11} L_{B O}$. These values are above the critical limit defined by Lehmer et al. (2007) and should therefore be considered optically bright.

Figure 2 shows the CMD of A2254 (B-R) vs $R$ for all galaxies. The early-type galaxies in the cluster are closely correlated with their optical colors and luminosity. This is known as the color-magnitude relationship or the red sequence. In general, galaxies significantly above the red sequence are in the cluster background (since they are redder than the reddest cluster galaxies). The number of red galaxies is significantly greater than the number of blue galaxies.

We analyzed GLF in band B using RTT150 data, to better understand the effects of cluster mergers on galaxy properties, we have seen that the GLF of the Abell 2254 can be placed in band $\mathrm{B}$ with a Schechter function. The best-fitting parameters of the Schechter function are the characteristic absolute magnitude in the $B$ band $M_{B}^{*}=19.67_{-0.96}^{+0.84} \mathrm{mag}$, and the slope at the faint end of the GLF band $\alpha=-1.29_{-0.34}^{+0.23} \mathrm{mag}$, for galaxies.

\section{Conclusions and Recommendations}

The main conclusions are as follows:

In our optical imaging studies, we have used the Bessel $B$, $V$, and $R$ filters, for the first time for A2254 (see Figure 1). All galaxies are listed in Table 2. The coordinates of member galaxies are found to be in very good agreement with the positions of the galaxies reported by Girardi et al. (2011).

A2254 should be considered as an optically bright galaxy since its $L_{B}$ range is above the critical limit defined by Lehmer et al. (2007). The number of red galaxies is significantly greater than the number of blue galaxies.

We have obtained the GLF in the $B$ band. It is shown that the GLF of Abell 2254 can be fitted well with a Schechter 438 
function in the $B$ band. The best-fitting parameters of the Schechter function are the characteristic absolute magnitude in the $B$ band $M_{B}^{*}=20.46_{-0.96}^{+0.84} \mathrm{mag}$, and the slope at the faint end of the GLF $\alpha=-1.29_{-0.34}^{+0.23}$ for galaxies.

Optical spectra of dominant galaxies in the cluster may be derived as a continuation of studies in the optical band of this cluster. The optical spectra will provide information about attributes of the cluster member galaxies and the optical profile of the host cluster.

\section{Acknowledge}

We thank TUBİTAK-TUG for their support in using RTT150 (Russian-Turkish Telescope) for our observations performed through Project number 15BRTT150-869.

\section{References}

Abell, G. O., Corwin R. P., \& Olowin, R. P. (1989). A Catalog of Rich Clusters of Galaxies. Astrophysical Journal Supplement,70, 1-138. https://doi.org/10.1086/191333

Adami, C., Durret, F., Mazure, A., Pello, R., Picat, J. P., West, M., \& Meneux, B. (2007). Spatial variations of the optical galaxy luminosity functions and red sequences in the Coma cluster: clues to its assembly history, Astronomy and Astrophysics, 462, 411-427. https://doi.org/10.1051/00046361:20065848

Andreon, S. (2001). Deep Near-Infrared Luminosity Function of a Cluster of Galaxies atz $=0.3$, The Astrophysical Journal, 547, 623-634. http://dx.doi.org/10.1086/318381

Barkhouse, W. A., Yee H. K. C., \& L'opez-Cruz O. (2007). The Red-Sequence Luminosity Function in Galaxy Clusters since $\mathrm{z} \sim 1, \quad$ The Astrophysical Journal, 671, 1471. https://doi.org/10.1086/524398

Baum, S. A. (1959). Population Inferences from Star Counts, Surface Brightness and Colors, Publications of the Astronomical Society of the Pacific, 71, 106-117. https://doi.org/10.1086/127346

Bertin, E., \& Arnouts, S. (1996). SExtractor: Software for source extraction, Astronomy and Astrophysics Supplement, 117, 393-404. https://doi.org/10.1051/aas:1996164

Blanton, E. L, Sarazin, C. L., \& Irwin, J. A. (2001). Diffuse Gas and Low-Mass X-Ray Binaries in the Chandra Observation of the S0 Galaxy NGC 1553. Astrophysical Journal, 552, 1, 106-119. https://doi.org/10.1086/320461

Boué, G., Adami, C., Durret, F., Mamon, G. A., \& Cayatte, V. (2008). The galaxy luminosity function of the Abell 496 cluster and its spatial variations, Astronomy \& Astrophysics, 479, 335-346. https://doi.org/10.1051/0004-6361:20077723

Caglar, T. \& Hudaverdi, M. (2017). XMM-Newton view of X ray overdensities from nearby galaxy clusters: the environmental dependencies, Monthly Notices of the Royal Astronomical Society, 471, 49905007. https://doi.org/10.1093/mnras/stx1811

Canizares, C. R., Fabbiano G., \& Trinchieri G. (1987). Properties of the X-Ray Emitting Gas in Early-Type Galaxies, Astrophysical Journal, 312, 503-513. https://doi.org/10.1086/164896

Crawford, C. S., Edge, A. C., Fabian, A. C., Allen, S. W., Bohringer, H., Ebeling, H., McMahon, R. G., \& Voges, W.
(1995). Optical spectroscopy of the ROSAT X-ray brightest clusters - II, Monthly Notices Royal Astronomical Society, 274, 75-84. https://doi.org/10.1093/mnras/274.1.75

De Propris, R., Colless, M., Driver, S. P., et al. (2003). The $2 \mathrm{dF}$ Galaxy Redshift Survey: the luminosity function of cluster galaxies, Monthly Notices of the Royal Astronomical Society, 342, 3, 725-737. https://doi.org/10.1046/j.13658711.2003.06510.x

Drory, N., Bender, R., Feulner, G., Hopp, U., Maraston, C., Snigula, J., \& Hill, G. J. (2003). The Munich Near-Infrared Cluster Survey. II. TheK-Band Luminosity Function of Field Galaxies toz 1.2, The Astrophysical Journal, 595, 698-711. http://dx.doi.org/10.1086/377497

Durret, F., Adami, C., Cappi,A., et al. (2011). Galaxy cluster searches based on photometric redshifts in the four CFHTLS Wide fields, Astronomy \& Astrophysics, 535, 65-80. https://doi.org/10.1051/0004-6361/201116985

Ebeling, H., Voges, W., Bohringer, H., Edge, A.C., Huchra J. P. \& Briel U. G. (1996). Properties of the X-ray-brightest Abell-type clusters of galaxies (XBACs) from ROSAT AllSky Survey data - I. The sample., Monthly Notices of the Royal Astronomical Society, 281, 799-829. https://doi.org/10.1093/mnras/281.3.799

Ellis, S. C., \& O'Sullivan E. (2006). Correlations of nearinfrared, optical and X-ray luminosity for early-type galaxies, Monthly Notices of the Royal Astronomical Society, 367, 627-645. https://doi.org/10.1111/j.13652966.2005.09982.x

Faber, S. M. (1973). Variations in Spectral-Energy Distributions and Absorption-Line Strengths among Elliptical Galaxies, Astrophysical Journal, 179, 731754. https://doi.org/10.1086/151912

Fabian, A.C. (1988). Theory of Intracluster Gas Hot Thin Plasmas in Astrophysics. Proceedings of a NATO Advanced Study Institute, held at Cargese, Corsica, September 8-18, 1987, Dordrecht: Kluwer, 1988, edited by R. Pallavicini. NATO Advanced Study Institute (ASI) Series C, 249, p.293.

Feng, L., Peng-fei, Y., \& Qi-rong Y. (2014). Luminosity Function of The Galaxy Cluster Abell 85, Chinese Astronomy and Astrophysics, Volume 38, Issue 3, p. $247-$ 256. https://doi.org/10.1016/j.chinastron.2014.07.003

Gallazzi, A., Charlot, S., Brinchmann J., \& White, S. D. M. (2006). Ages and metallicities of early-type galaxies in the Sloan Digital Sky Survey: new insight into the physical origin of the colour-magnitude and the $\mathrm{Mg} 2-\sigma \mathrm{V}$ relations, Monthly Notices of the Royal Astronomical Society, 370, 1106-1124. https://doi.org/10.1111/j.13652966.2006.10548.x

Gilfanov, M. (2004). Low-mass X-ray binaries as a stellar-mass indicator for the host galaxy, Monthly Notices of the Royal Astronomical Society, 349, 146-168. https://doi.org/10.1111/j.1365-2966.2004.07473.x

Girardi, M., Bardelli, S., Barrena, R., Boschin, W., Gastaldello, F., \& Nonino M. (2011). Internal dynamics of Abell 2254: a merging galaxy cluster with a clumpy, diffuse radio emission. Astronomy \& Astrophysics, 536, 89109. https://doi.org/10.1051/0004-6361/201117332

Girardi, M., Barrena, R., \& Boschin, W. (2012). Merging clusters of the DARC sample: studying the formation of galaxy clusters. in "Growing-up at High Redshift: From Proto-clusters to Galaxy Clusters". Posters from the Conference held in Sept (2012) Madrid-Spain. 
http://www.sciops.esa.int/index.php?project=CONF2011\&pa ge $=$ CLUSTERS2012

Kodama, T., \& Arimoto, N. (1997). Origin of the colourmagnitude relation of elliptical galaxies, Astronomy and Astrophysics, 320, 41-53.

Kodama, T., Bower, R. G., \& Bell, E. F. (1999). The colour \pm magnitude relation of early-type galaxies in the Hubble Deep Field, Monthly Notices of the Royal Astronomical Society, 306, 561566. https://doi.org/10.1046/j.1365-8711.1999.02542.x

Lehmer, B. D., Brandt, W. N., Alexander, D. M., Bell, E. F., Mc Intosh D. H., Bauer, F. E., Hasinger, G., Mainieri V., Miyaji, T., Schneider, D. P. \& Steffen, A. T. (2007). The X-Ray Evolution of Early-Type Galaxies in the Extended Chandra Deep Field-South. The Astrophysical Journal, 657, 681-699. https://doi.org/10.1086/511297

Mahdavi, A. \& Geller, M. J. (2001). The LX- $\sigma$ Relation for Galaxies and Clusters of Galaxies, The Astrophysical Journal, 554, 129-132. https://doi.org/10.1086/321710

Massarotti, M. Busarello, G., La Barbera, F., \& Merluzzi, P. (2003). UV-NIR restframe luminosity functions of the galaxy cluster EIS 0048 at $\mathrm{z} \sim 0.64$, Astronomy \& Astrophysics, 404, 75-81. https://doi.org/10.1051/00046361:20030473

Monet, D., et al. (1998). VizieR Online Data Catalog: A catalogue of astrometric standards. CDS/ADC Collection of Electronic Catalogues, 1252, 0.

O'Sullivan, E., Forbes, D. A., \& Ponman, T. J. (2001). A catalogue and analysis of X-ray luminosities of early-type galaxies, Monthly Notices of the Royal Astronomical Society, 328, 461-484. https://doi.org/10.1046/j.13658711.2001.04890.x

Press, W. H., \& Schechter P. (1974). Formation of Galaxies and Clusters of Galaxies by Self-Similar Gravitational Condensation Show affiliations, Astrophysical Journal, 187, 425-435, https://doi.org/10.1086/152650

Randall, S. W., Sarazin, C. L., \& Irwin, J. A. (2006). XMMNewton Observation of Diffuse Gas and Low-Mass X-Ray Binaries in the Elliptical Galaxy NGC 4649 (M60). Astrophysical Journal, 636, 1, 200-213. https://doi.org/10.1086/497977

Sarazin, C.L. (1986). X-ray emission from clusters of galaxies. Reviews of Modern Physics. 58, 1-116 https://doi.org/10.1103/RevModPhys.58.1.

Sarazin, C.L. (1988). X-Ray Emissions from Clusters of Galaxies. Cambridge: Cambridge Univ. Press. 252 pp.

Sarazin, C.L. (1992). The Intracluster Medium ed. A. C. Fabian (Dordrecht: Kluwer), 131-151. https://doi.org/10.1007/97894-011-2482-9

Schechter, P. (1976). An analytic expression for the luminosity function for galaxies., Astrophysical Journal, 203, 297306. https://doi.org/10.1086/154079

Schindler, S., \& Müller, E. (1993). Simulations of the evolution of galaxy clusters. II. Dynamics of the intra-cluster gas. Astronomy and Astrophysics, 272, 137-152.

Smith, R. K. Brickhouse, N. S., Liedahl, D. A. \& Raymond, J. C. (2001). Collisional Plasma Models with APEC/APED: emission-line Diagnostics of Hydrogen-like and Helium-like Ions, The Astrophysical Journal, 556, 91-95. https://doi.org/10.1086/322992

Struble, M. F., \& Rood, H. J. (1987). A Compilation of Redshifts and Velocity Dispersions for Abell Clusters, Astrophysical
Journal Supplement,
https://doi.org/10.1086/191173

Worthey G. (1994). Comprehensive Stellar Population Models and the Disentanglement of Age and Metallicity Effects, Astrophysical Journal Supplement, 95, 107 149. https://doi.org/10.1086/192096 